\title{
Análisis preliminar de la vulnerabilidad física ante desbordes en la zona de influencia de la quebrada río Seco de la ciudad de Huaraz en el año 2015
}

\author{
Preliminary analysis of the physical vulnerability to overflows in the zone of \\ influence of the ravine rio Seco of the city of Huaraz in the year 2015 \\ Marco Silva Lindo ${ }^{1}$, Reynaldo Reyes Roque ${ }^{1}$, Rosa Rodríguez Anaya ${ }^{1}$ y \\ Grimaldo Jara Palmadera ${ }^{1}$
}

\section{RESUMEN}

La ciudad de Huaraz se ubica en una zona expuesta a diversos peligros, resultado de su configuración y ubicación geográfica. Muchos de estos fenómenos pueden devenir en desastres que pueden afectar sobremanera la actividad socioeconómica de la región y del país. Un sector característico de Huaraz, es el llamado río Seco, que es un curso hidrográfico ubicado al sur de la ciudad, expuesto a desbordes por inundaciones y que ha tenido un fuerte crecimiento en los últimos años. En este sentido, el estudio se planteó determinar la vulnerabilidad física de las viviendas de la zona de influencia de la quebrada ante desbordes del río Seco de la ciudad de Huaraz. La metodología adoptada fue de investigación cuantitativa no experimental y transversal, analizando el fenómeno en su situación en el año 2015. La metodología consistió en el levantamiento topográfico del terreno, obtención de información pluviométrica e identificación de las características de 582 viviendas ubicadas en el margen del río, para determinar posteriormente su nivel de vulnerabilidad ante desbordes. Finalmente, se determinó el nivel del peligro de desbordes en la zona del río Seco y se obtuvo que el 57.9\% de las viviendas tiene un nivel alto de vulnerabilidad.

Palabras clave: vulnerabilidad; vulnerabilidad física; desbordes de río.

\section{ABSTRACT}

The Huaraz city is located in an area exposed to different hazards, result of its configuration and geographical location. Many of these phenomena can become disasters that can greatly affect the socio-economic activity of the region and the

1 Universidad Nacional del Santa. Chimbote, Perú. 
country. One characteristic of Huaraz area, is the so-called Rio Seco, is a hydrographic course located to the south of the city, exposed to overflow by flooding and that has had a strong growth in recent years. In this sense the study arose determine physical vulnerability to overflow of dwellings in the influence area of the Rio Seco ravine in Huaraz city. The methodology adopted was quantitative research non-experimental and cross, analyzing the phenomenon in its situation by the year 2015. The methodology consisted of topographic survey of the terrain, pluviometric information obtaining and identification of the characteristics of 582 houses located on the bank of the River, to later determine their level of vulnerability to overflow.

Finally determined the level of danger of overflow in the zone of the rio Seco was obtained to the $57.9 \%$ of dwellings have a high level of vulnerability.

Keywords: vulnerability; physical vulnerability; river overflow

\section{ICHIKLLACHAW}

Waras marka karanqanqa mantsanaypaqmi rahukuna chakinllachaw kanqanraykur. Kay mantsay llakikuyqa llapan kay suyuchaw taakuq runakunapaqmi allitsu kanman. Kananqa Waraspa llakikuyninqa Tsaki Mayun kapunqanmi, kayqa Waraspa qullan kaqninchawmi karan, allaapa tamya kaptinqa apaakuynawmi aykamunman. Tsayraykurmi kay musyapakuy riqimantsik kay Tsaki Mayu imanawmi Waraschaw rurashqa wayikunata itsapis huchuynin mantsanaypaqmi kanman. Kay musyapakuytaqa kwantitatiwawan mana ikpirimintal nishqanwanmi rurakashqa. 2015 watachaw imanaw kay kanqan. Mituduluhiyawan Waraspa imanaw patsan kanqantam pallarikashqa, tsay willakuyninchawqa 582 mayupa kuchunchawmi wayikuna shaarikashqantam riqitsikun, tsaywan patsaypa matsay kanqantam riqitsikun.

Tsay llapanwanqa riqitsimantsik maykamash kay Tsaki Mayupa mantsay llakikuynin kanqantam, tsaypiq 57.9\% tum wayikunam matsay llakikuychaw kayan.

Pushaq shimikuna: mantsay llakikuynin; wayi huchuy mantsaynin; mayupa apaakuynin.

\section{INTRODUCCIÓN}

Este trabajo tiene como propósito contribuir al desarrollo social de zonas en riesgo con la determinación de los factores que las hacen vulnerables, en beneficio de pobladores que viven en sectores pobres y deprimidos. En Ancash, una de las provincias más afectadas por los desastres es la de Huaraz, amenazada principalmente por sismos, aluviones e inundaciones (Chuquisengo, 2007). Según el SINAGERD (2014), Huaraz ha sido una de las ciudades más afectadas en el país por aluviones, a causa de desbordes de lagunas, como el del 13 de diciembre de 1941, que causó la muerte de alrededor del 
30\% de la población, y por sismos como el del 31 de mayo de 1970, de magnitud 7,8 grados en la escala de Richter, que habría producido la pérdida de aproximadamente 25,000 vidas humanas.

La ciudad de Huaraz experimenta un incremento sostenido de sus habitantes y del número de viviendas, pues en 26 años prácticamente se ha triplicado. Ferro (2006), determina el riesgo de desastre de la ciudad de Huaraz mediante el análisis del desarrollo urbano y la creación de las condiciones de riesgo; estima que este patrón de crecimiento responde a las precarias condiciones sociales y económicas de gran parte de la población, las que contribuyen a que los nuevos hogares ocupen predios en áreas marginales muy expuestas o hacinen predios en áreas consolidadas, buscando no perder el acceso a oportunidades económicas o de superación.

Un sector vulnerable de la ciudad de Huaraz, es el llamado río Seco, denominado así porque solo tiene discurrimiento de aguas en las estaciones de lluvias; ubicado al sur de la ciudad, descarga sus aguas al río Santa, cuya zona de influencia ha sufrido un crecimiento desordenado de viviendas. Según Soluciones Prácticas (2012), ocurre el fenómeno de colmatación a lo largo del cauce del río por el importante volumen de material arrastrado en estaciones de lluvias. El peligro generado por el transporte de sedimentos en el río Seco se da aguas abajo en su entrega al río Santa, pues su cono de deyección se ha visto invadido por un asentamiento humano, el mismo que puede ser afectado si hay un aumento considerable del material arrastrado por lluvias extraordinarias. La zona de estudio se presenta en la figura 1.

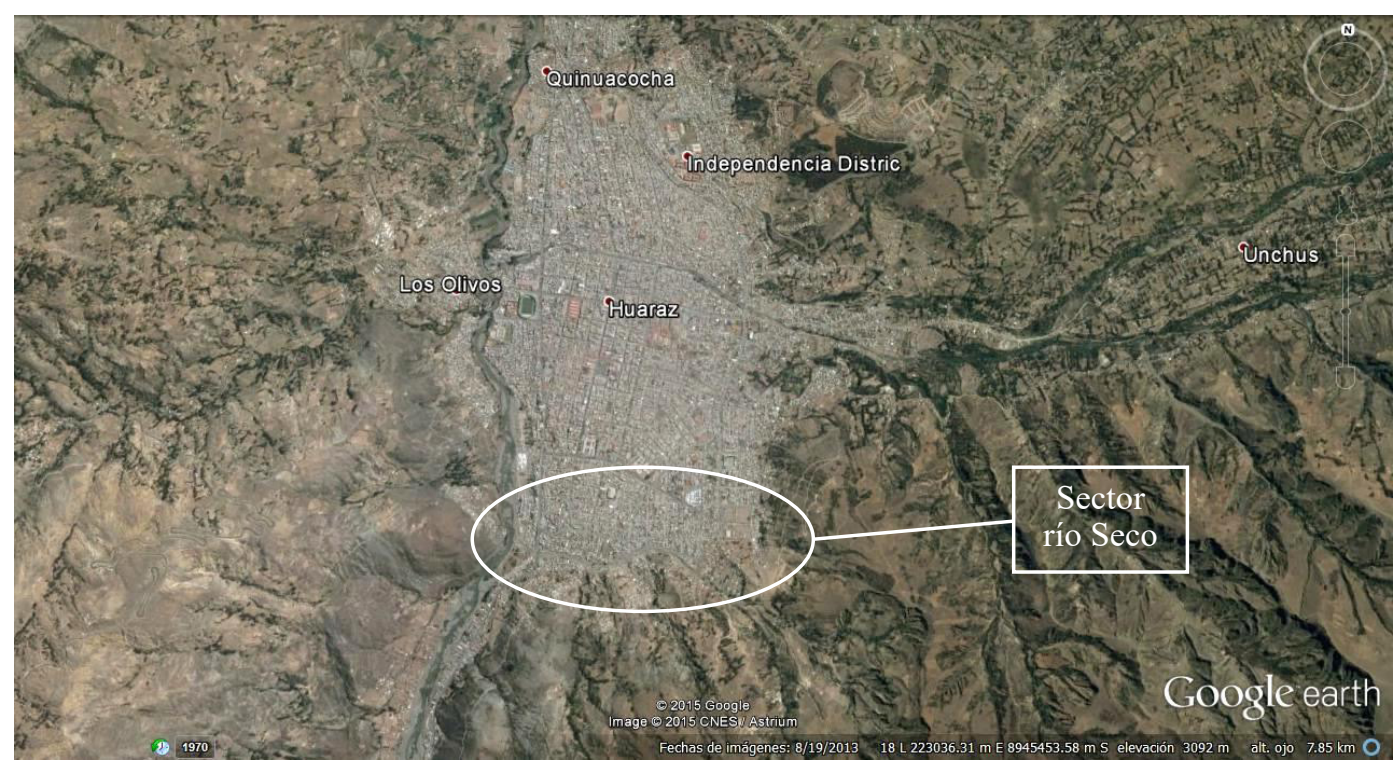

Figura 1. Ubicación del sector río Seco en la ciudad de Huaraz. Fuente: Google Earth 
El peligro de una inundación o desborde es el resultado de la combinación de probabilidad e intensidad (Somos-Valenzuela et al, 2014) debido a la presencia de grandes cantidades de agua en muy corto espacio de tiempo. La evaluación del peligro de desbordes consiste en definir los niveles de susceptibilidad a desbordes por crecidas del río, que involucra la estimación de los picos de las descargas o caudales, incluyendo el nivel que alcanza y la zonificación de áreas susceptibles en caso de crecidas extraordinarias. Para la estimación de caudales máximos, se acude a métodos alternativos, basados la mayoría de ellos en datos de precipitación máxima en 24 horas y en las características físicas de la cuenca, para así inferir los caudales máximos. El método más usual para estimación de caudales es la Fórmula o Método Racional (MTC 2008), muy usado para cuencas pequeñas con áreas máximas entre 10 o $20 \mathrm{Km}^{2}$, que considera un coeficiente de escorrentía que depende de factores del terreno (Calle y Rodríguez, 2013); la intensidad de precipitación máxima horaria asociada al período de retorno seleccionado y a una duración igual al tiempo de concentración de la cuenca pluvial, y el área aportante.

Según el Instituto Nacional de Defensa Civil (2006), la vulnerabilidad es el grado de debilidad o exposición de un elemento o conjunto de elementos frente a la ocurrencia de un peligro natural o antrópico de una magnitud dada. La vulnerabilidad está íntimamente ligada a los procesos sociales que se desarrollan en las áreas propensas y usualmente tiene que ver con la fragilidad, la susceptibilidad o la falta de resiliencia de la población ante amenazas de diferente índole (BID, 2010). La vulnerabilidad física se refiere especialmente a la localización de los asentamientos humanos en zonas de riesgo, y a las deficiencias de las estructuras físicas para absorber los efectos de los peligros (DGOA, 2012). En inundaciones, explica Kuroiwa (2002) que la vulnerabilidad física depende de la resistencia de las edificaciones para tomar presiones hidrostáticas e hidrodinámicas, su flotabilidad y la resistencia de su cimentación al efecto erosivo de las aguas; también depende de la estabilidad de los materiales de construcción para permanecer inmersos bajo agua por algún tiempo. En el análisis de vulnerabilidad, según SNGR (2011), el propósito es evaluar, en forma cualitativa la vulnerabilidad física estructural de edificaciones, basado en una calificación de las características de las estructuras frente a amenazas de desbordes. Para este análisis es fundamental determinar el aporte a la vulnerabilidad generada por cada uno de los elementos estructurales (variables de vulnerabilidad) de la edificación, para estimar el nivel de vulnerabilidad de la vivienda ante la amenaza de desborde.

Se justifica el estudio por cuanto trata de la determinación de factores que hacen vulnerable a un sector y grupo humano importante de la ciudad de Huaraz, que ve limitadas sus aspiraciones de desarrollo por la incertidumbre de los peligros a los que pueda estar expuesto, cuyos resultados brindarán elementos de juicio a las autoridades locales para iniciar una gestión del desarrollo local, que lleve a Huaraz a convertirse en una ciudad sostenible y segura. En ese sentido, el objetivo del estudio fue determinar de manera preliminar la vulnerabilidad física de las viviendas ante desbordes de la zona de influencia de la quebrada río Seco de la ciudad de Huaraz. 
A continuación se presenta la metodología seguida para la estimación del peligro de desbordes en la zona de influencia de la quebrada río Seco en el año 2015, con la determinación de las áreas susceptibles por caudales debidos a precipitaciones máximas en la cuenca aportante, para finalmente determinar la vulnerabilidad física de las edificaciones en función de las características de su sistema estructural, con la preparación de los planos de peligro de inundación y de vulnerabilidad física de las viviendas.

\section{MATERIALES Y MÉTODOS}

Se trata de una investigación cuantitativa no experimental y transversal, que analiza el fenómeno en su situación en el año 2015. La población la constituyen los asentamientos en la zona de influencia del río Seco en la ciudad de Huaraz, Región Ancash, Perú. Se consideró como viviendas de la zona de influencia del río Seco, a aquellas ubicadas hasta $100 \mathrm{~m}$ del eje del río, lo que fue corroborado con el levantamiento topográfico de la microcuenca desde su intersección con la calle Jr. Ricardo Palma hasta su encuentro con el río Santa, en una longitud aproximada de $1.95 \mathrm{~km}$. La población está dada aproximadamente por 604 viviendas. La unidad de análisis es la formada por las viviendas de los asentamientos en la zona de influencia del río Seco en la ciudad de Huaraz. Se determinó una muestra estadística confiable resultante de 582 viviendas con encuestas válidas para el procesamiento.

La metodología, en el trabajo de campo, partió de la revisión de los instrumentos diversos para la recopilación de datos, la delimitación de la microcuenca, el levantamiento topográfico del área de estudio, obtención de datos pluviométricos de la estación de meteorología «Santiago Antúnez de Mayolo», así como de la identificación de las características de las viviendas que se encuentran ubicadas en el margen del río Seco a través de una ficha técnica, para estimar el peligro de desborde en la zona de influencia del río Seco y determinar posteriormente el nivel de vulnerabilidad de las viviendas frente a desbordes.

La metodología, en relación a la estimación del peligro de desbordes de la zona ribereña del río Seco, obedece a procedimientos estándares de métodos cuantitativos de la gestión del riesgo de desastres para la determinación de las áreas susceptibles a esta amenaza. Para el cálculo de caudales se realizó el análisis de frecuencias de eventos hidrológicos máximos, aplicables a precipitación máxima de 24 aplicando el método racional. Para determinar la magnitud del peligro de desbordes del río Seco en la ciudad de Huaraz en el año 2015, después de realizar el modelamiento en el programa HEC-RAS 4.1.0, se procedió a procesar la información en el programa Hec Georas que es extensión del ArcGIS, y se obtuvo el plano de inundación para diferentes períodos de retorno, denominado Plano de Peligro de inundación ante desbordes en el sector de río Seco. La franja del peligro de inundación se realizó con caudales para el período de retorno de $\mathrm{T}=75$ años (Peligro Muy Alto), para $\mathrm{T}=100$ años (Peligro Alto), para $\mathrm{T}=200$ (Peligro Medio) y $\mathrm{T}=500$ años (Peligro Bajo). 
La metodología en relación a la vulnerabilidad física de las edificaciones consistió en la aplicación del método de la Guía para implementar el análisis de vulnerabilidad a nivel de cantonal, de la Secretaría Nacional de Gestión de Riesgos del Ecuador (SNGR, 2011). Se elaboró una ficha técnica para tomar nota de las características del sistema estructural de cada una de las edificaciones, considerando las variables de vulnerabilidad señaladas en la Tabla 1. Se comprobó los datos obtenidos de la ficha técnica, verificando que la ubicación de las edificaciones sea compatible con el plano catastral y cuidando que la información tenga una completitud de datos superior al 90\%. Se calificó cada elemento estructural del predio conforme a lo establecido en la tabla. Cada variable de vulnerabilidad estructural tiene un valor, frente a la amenaza de desborde. La menor vulnerabilidad se califica con 1 y la mayor vulnerabilidad con 4. Una vez calificada cada una de las variables de vulnerabilidad, se realiza una ponderación de las variables con relación a la amenaza que se está evaluando.

Tabla 1. Ponderación de los variables de vulnerabilidad ante el peligro de desbordes

\begin{tabular}{|c|c|c|c|c|c|c|}
\hline \multirow{2}{*}{$\begin{array}{l}\text { VARIABLE DE VULNERABILIDAD } \\
\text { Sistema estructural }\end{array}$} & \multicolumn{4}{|c|}{$\begin{array}{l}\text { VALORES PO- } \\
\text { SIBLES DEL } \\
\text { INDICADOR }\end{array}$} & \multirow{2}{*}{$\begin{array}{l}\text { PONDE- } \\
\text { RACIÓN } \\
3\end{array}$} & \multirow{2}{*}{$\begin{array}{c}\text { VALOR } \\
\text { MÁXIMO } \\
12\end{array}$} \\
\hline & 4 & 3 & 2 & 1 & & \\
\hline Tipo de material en paredes & 4 & 3 & 2 & 1 & 3 & 12 \\
\hline Tipo de cubierta & 3 & 2 & 1 & & 1 & 3 \\
\hline Sistema de entrepisos & 4 & 3 & 2 & 1 & 2 & 8 \\
\hline Número de pisos & 4 & 3 & 2 & 1 & 2 & 8 \\
\hline Características del suelo bajo la edificación & 4 & 3 & 2 & 1 & 3 & 12 \\
\hline Antigüedad de la edificación & 4 & 3 & 2 & 1 & 2 & 8 \\
\hline Topografía del terreno de la vivienda & 4 & 3 & 2 & 1 & 4 & 16 \\
\hline Configuración geométrica en planta & 4 & & & 1 & 1.25 & 5 \\
\hline Existe concentración de masas en nivel & 4 & & & 1 & 1 & 4 \\
\hline $\begin{array}{l}\text { Otros factores que incidan en la vulnerabi- } \\
\text { lidad }\end{array}$ & 4 & & & 1 & 2 & 8 \\
\hline \multirow[t]{2}{*}{ Distancia de la vivienda al margen de río } & 4 & 3 & 2 & 1 & 1 & 4 \\
\hline & \multicolumn{5}{|c|}{ Valor Mínimo $=25.25$} & 100 \\
\hline
\end{tabular}

Fuente: Elaborado en base a la Guía para implementar el análisis de vulnerabilidad a nivel cantonal, de la Secretaría Nacional de Gestión de Riesgos (SNGR, 2011)

Se procedió a calificar cada vivienda en función de la cantidad de puntos obtenidos, asignándoles un nivel de vulnerabilidad de acuerdo a la tabla siguiente. 
Tabla 2. Rangos de calificación del nivel de vulnerabilidad de las edificaciones

\begin{tabular}{lc}
\hline Nivel del Vulnerabilidad & Puntaje \\
\hline Baja & 25.25 a 33.2 \\
Media & 33.3 a 59.5 \\
Alta & 59.6 a 88.5 \\
Muy alta & 88.6 a 100 \\
\hline
\end{tabular}

Con la información de las fichas técnicas, se preparó un mapa mostrando las edificaciones con su correspondiente nivel de vulnerabilidad ante desbordes en el sector río Seco.

\section{RESULTADOS}

Las viviendas en la zona ribereña del río Seco predominantemente son de albañilería confinada $(48.46 \%)$ y de adobe $(41.67 \%)$, como se muestra a continuación.

Tabla 3. Distribución de viviendas analizadas según su sistema estructural

\begin{tabular}{lr}
\hline Sistema estructural & Porcentaje \\
\hline Adobe & $41.67 \%$ \\
Quincha & $1.54 \%$ \\
Mampostería & $0.66 \%$ \\
Estructura de madera & $0.44 \%$ \\
Adobe reforzado & $0.22 \%$ \\
Albañilería & $4.17 \%$ \\
Albañilería confinada & $48.46 \%$ \\
Concreto armado & $2.85 \%$ \\
TOTAL & $100.00 \%$ \\
\hline
\end{tabular}

Peligro de desbordes en la zona de influencia de la quebrada río Seco

Se determinó la franja de inundación en el sector de río Seco, donde se identificó los niveles de peligro ante desbordes mediante franjas de color: rojo para el nivel muy alto, naranja para el nivel alto, amarillo para el nivel medio y verde para el nivel bajo. En el anexo se muestra el plano de peligro de desbordes para la zona de río Seco en Huaraz, para el año 2015. 
Nivel de vulnerabilidad ante desbordes de las viviendas en el sector río Seco

Se calificó cada vivienda en función de la cantidad de puntos obtenidos, asignándoles un nivel de vulnerabilidad de acuerdo a la tabla 2 y se preparó un mapa, que se muestra en el anexo, visualizando todas las edificaciones con su correspondiente nivel de vulnerabilidad ante desbordes en el sector río Seco. El porcentaje de viviendas por nivel de vulnerabilidad física se muestra en la siguiente tabla, donde se aprecia que la mayor parte de edificaciones del sector de río Seco presenta una vulnerabilidad alta, representando el $57.2 \%$.

Tabla 4. Porcentaje de viviendas por nivel de vulnerabilidad física en el sector de río Seco

\begin{tabular}{lcc}
\hline $\begin{array}{c}\text { NIVEL DE VULNE- } \\
\text { RABILIDAD }\end{array}$ & $\begin{array}{c}\text { NUMERO DE VI- } \\
\text { VIENDAS }\end{array}$ & $\begin{array}{c}\text { PORCENTAJE DE VI- } \\
\text { VIENDAS }\end{array}$ \\
\hline Muy alta & 4 & $0.7 \%$ \\
Alta & 333 & $57.2 \%$ \\
Media & 238 & $40.9 \%$ \\
Baja & 7 & $1.2 \%$ \\
TOTAL & 582 & $100.0 \%$ \\
\hline
\end{tabular}

El porcentaje de aporte de cada una de los variables a la construcción de la vulnerabilidad física estructural de la vivienda se muestra en la tabla siguiente.

Tabla 5. Porcentaje de aporte a la vulnerabilidad de las variables estructurales de las viviendas del sector río Seco

\begin{tabular}{lcccc}
\hline \multicolumn{1}{c}{ VARIABLES ESTRUCTURALES } & \multicolumn{5}{c}{ VALORES } \\
& 4 & 3 & 2 & 1 \\
\hline Sistema estructural & $45.5 \%$ & $18.0 \%$ & $16.8 \%$ & $19.6 \%$ \\
Tipo de material en paredes & $45.4 \%$ & $0.0 \%$ & $0.2 \%$ & $54.1 \%$ \\
Tipo de cubierta & $0.0 \%$ & $45.0 \%$ & $3.4 \%$ & $50.2 \%$ \\
Sistema de entrepisos & $7.7 \%$ & $16.7 \%$ & $0.3 \%$ & $48.6 \%$ \\
Número de pisos & $2.4 \%$ & $12.9 \%$ & $46.0 \%$ & $38.3 \%$ \\
Características del suelo bajo la edificación & $33.2 \%$ & $36.6 \%$ & $28.0 \%$ & $2.1 \%$ \\
Antigüedad de la edificación & $0.5 \%$ & $11.0 \%$ & $82.0 \%$ & $4.5 \%$ \\
Topografía del terreno de la vivienda & $9.5 \%$ & $6.2 \%$ & $38.0 \%$ & $46.2 \%$ \\
Configuración geométrica en planta & $12.0 \%$ & $0.0 \%$ & $0.0 \%$ & $88.0 \%$ \\
Existe concentración de masas en nivel & $8.1 \%$ & $0.0 \%$ & $0.0 \%$ & $91.2 \%$ \\
Otros factores que incidan en la vulnerabili- & $71.1 \%$ & $0.0 \%$ & $0.0 \%$ & $28.7 \%$ \\
dad & & & & \\
Distancia de la vivienda al margen del río & $58.8 \%$ & $19.6 \%$ & $13.7 \%$ & $7.4 \%$ \\
\hline
\end{tabular}




\section{DISCUSIÓN}

El análisis de la franja de inundación en el sector río Seco, nos lleva a establecer hasta cinco tramos considerados críticos, por donde se presentaría el desborde del río, y se ubica en las progresivas $0+170,0+295,0+375,0+570$ y $0+725$.

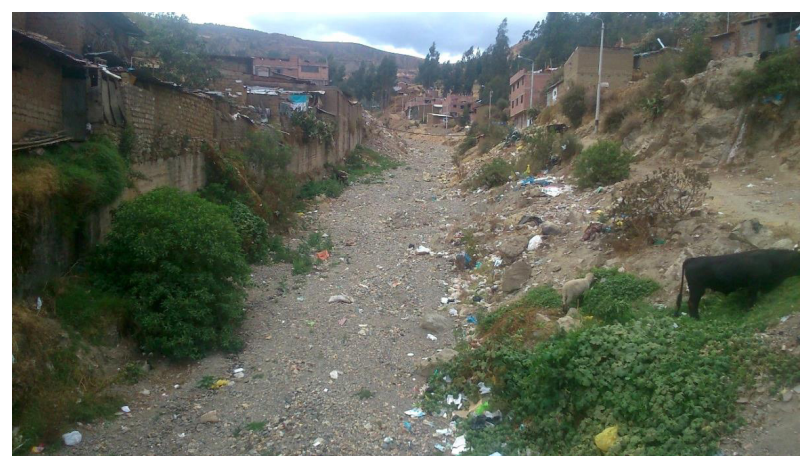

Figura 2: Sector crítico en la microcuenca río Seco

Si agrupamos las viviendas con vulnerabilidad muy alta y vulnerabilidad alta, se obtiene que el 57.9\% de las viviendas ubicadas en la zona de influencia del río Seco, tiene una vulnerabilidad igual o mayor a la vulnerabilidad Alta ante desbordes de este río. Por otro lado, se aprecia que solamente el 1.2\% de las viviendas tiene una vulnerabilidad baja ante desbordes.

La gráfica de barras representa el porcentaje de aporte de cada una de los variables a la construcción de la vulnerabilidad física estructural de la vivienda. La calificación de 1 significa que no aporta significativamente a la vulnerabilidad y la calificación 4 implica que la variable incrementa de forma importante la vulnerabilidad ante la amenaza de desbordes.

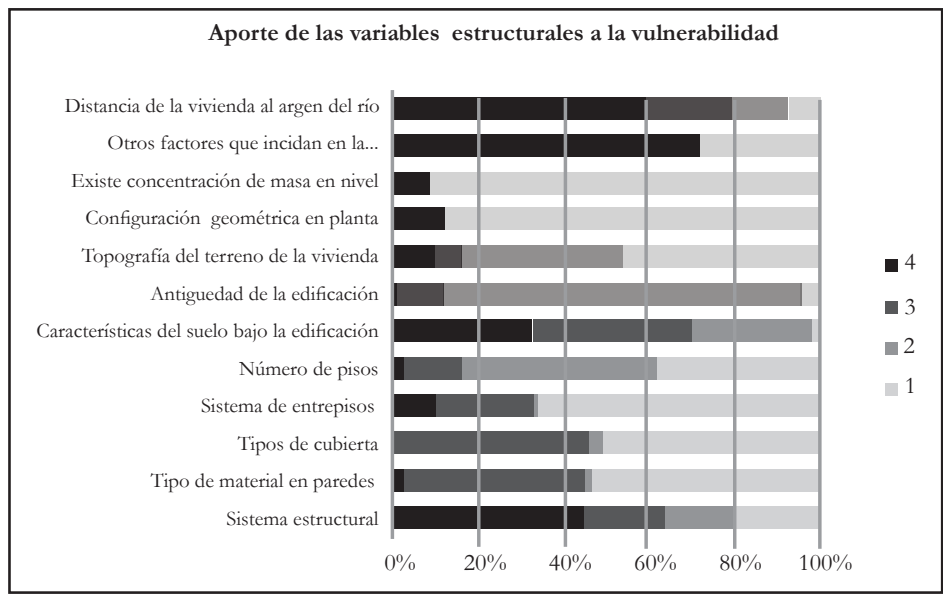

Figura 3. Porcentaje de aporte a la vulnerabilidad ante desbordes de las variables estructurales de las viviendas del sector río Seco 
Se puede identificar las variables que mayor influencia tienen en la vulnerabilidad: el sistema estructural, el tipo de material en las paredes, otros factores (como humedad, colapso de elementos, cargas laterales) y distancia al margen del río, pues a más del $40 \%$ de los predios los han calificado con 4 puntos. Lo que implica que las viviendas, con estas características, requieren de una atención especial tanto para enmarcarlas dentro de planes de mitigación como para realizar estudios de mayor especificidad para determinar la resistencia real de la estructura ante la amenaza de desbordes.

\section{CONCLUSIONES}

Las viviendas en zona ribereña del río Seco predominantemente son de albañilería confinada y de adobe, que significan el $48.46 \%$ y el $41.67 \%$, respectivamente.

Los tramos considerados críticos son cinco, por donde se presentaría el desborde del río Seco, y se ubican en las progresivas siguientes $0+170,0+295,0+375,0+570$ y $0+725$.

Se estima que el 57.9\% de las viviendas en la zona del río Seco en el distrito de Huaraz, presenta un nivel mayor a la vulnerabilidad alta, y solo el 1.2\% de las viviendas presenta un nivel de vulnerabilidad baja al peligro de desbordes.

Las variables que mayor influencia tienen en la vulnerabilidad ante desbordes de las viviendas en la zona del río Seco, son el sistema estructural, el tipo de material en las paredes, otros factores (como humedad, colapso de elementos, cargas laterales) y distancia al margen del río.

Las medidas estructurales de mitigación necesarias son la construcción de muros de contención en los puntos críticos identificados y la descolmatación periódica del cauce del río, así como la reubicación de los predios de las zonas muy expuestas y que recién se están consolidando.

Entre las medidas no estructurales de mitigación se destaca que el gobierno local debe mejorar el control de la ocupación del espacio urbano y de la construcción de las edificaciones, para restringir la ocupación urbana de sectores de la ciudad calificados como de alto peligro y que se construyan con deficiencias estructurales que aumenten su vulnerabilidad. Por otro lado, debe evitarse que se deposite basura o escombros en el cauce del río.

En este proyecto de carácter preliminar, el modelamiento del área de inundación se realizó considerando solo el flujo del agua, siendo preciso profundizar el análisis con el modelamiento hidráulico del río Seco considerando el transporte de sólidos y sedimentos, además de profundizar la evaluación de los sectores determinados con alta vulnerabilidad. 


\section{REFERENCIAS BIBLIOGRÁFICAS}

BID. 2010. Indicadores de Riesgo de Desastre y de Gestión de Riesgos. Programa para América Latina y el Caribe. Panamá: Banco Interamericano de Desarrollo. División de Medio Ambiente, Desarrollo Rural y Gestión del Riesgo de Desastres.

Calle, Franklin y Rodríguez, Byron. 2013. Diseño integral del sistema de drenaje vial para obras de arte menor (drenaje longitudinal y trasversal) para la carretera Pachón Mina Zharo de 7.2 km ubicada en el Cantón Suscal en la provincia de Cañar. Cuenca: Tesis de pre grado. Universidad de Cuenca. Ecuador.

Chuquisengo, Orlando. 2007. Gestión de riesgos en Ancash. Experiencias y propuestas. Lima: Soluciones Prácticas ITDG.

DGOA. 2012. Metodología para el Análisis de la Vulnerabilidad. San Salvador: Dirección General del Observatorio Ambiental, Unidad de Estudios Territoriales.

Ferro, Vladimir. 2006. La evaluación del riesgo de desastre en la planificación del desarrollo de la ciudad de Huaraz, Áncash, 2006. Lima: Tesis de maestría. Programa de Maestría en Gestión de Desastres para el Desarrollo Sostenible de la Facultad de Ingeniería Civil de la Universidad Nacional de Ingeniería.

Instituto Nacional de Defensa Civil. 2006. Manual Básico para la Estimación del Riesgo. Lima: Dirección Nacional de Prevención: Unidad de Estudios y Evaluación de Riesgos.

Kuroiwa, Julio. 2002. Reducción de Desastres: Viviendo en armonía con la Naturaleza. Lima: Quebecor World Perú S.A. Primera edición.

MTC. 2008. Manual de Hidrología, Hidráulica y Drenaje PERÚ. Lima: Ministerio de Transportes y Comunicaciones.

SINAGERD. 2014. Actividades prioritarias de Gestión del Riesgo de Desastres 2015-2016. Lima: Con Buena Letra Impresiones SRL.

SNGR. 2011. Guía para implementar el Análisis de Vulnerabilidad a Nivel Cantonal. Quito: Secretaría Nacional de Gestión de Riesgos.

Soluciones Prácticas. 2012. Análisis de Riesgo de Desastres Microcuenca del Rio Quillcay Paria, Distritos de Independencia y Huarar, Ancash, Perú. Lima: Soluciones Prácticas.

Somos-Valenzuela, Marcelo et al. 2014. Modelo de Inundación por potencial aluvión desde la Laguna Palcacocha, Huaraz, Perú. Lima: Corporación Globalmark.

Fecha de recepción: 7 de setiembre de 2016

Fecha de aceptación: 9 de diciembre de 2016

\section{Correspondencia}

Marco Silva Lindo

maslindo1@yahoo.com 
Anexo A. Plano de peligro de inundación y Plano de nivel de vulnerabilidad física de las viviendas ante desbordes en la zona de Rio Seco, año 2015
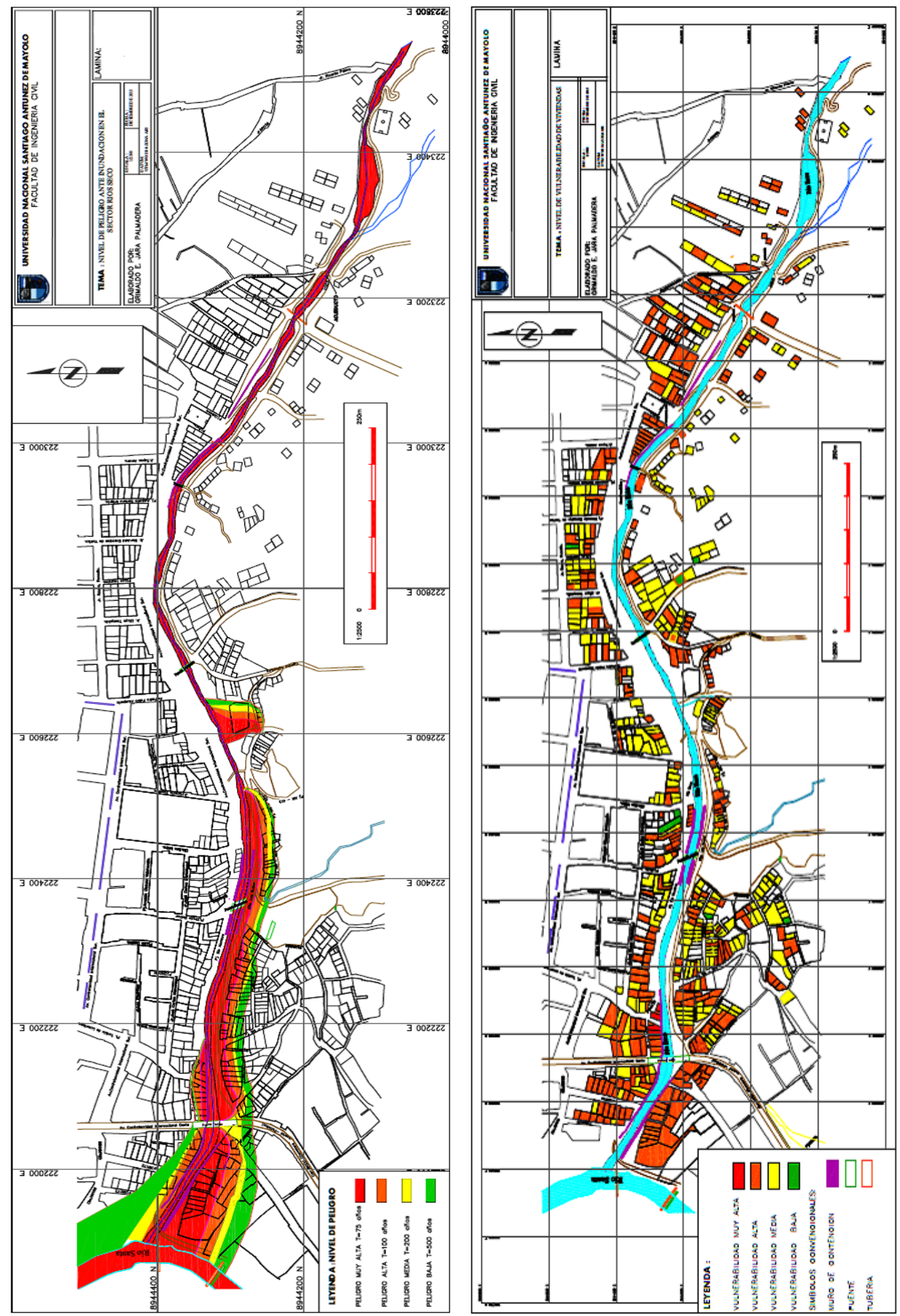